\title{
Una estrategia de desarrollo sostenible para Xalapa
}

Fortuno Hernández, Josefa Carolina

Universidad Veracruzana, México jfortuno@uv.mx
Hákim Krayem, Mónica Rubiette

Universidad Veracruzana, México rhakim@uv.mx
Resumen - Para considerar las bases creativas y culturales de la ciudad de Xalapa, Veracruz y sea reconocida como una Ciudad Creativa de la Música (UNESCO), proponemos una estrategia de desarrollo donde participen gobierno, empresas y sociedad para formar una industria creativa de la música.

Mostramos el contexto económico, social y cultural de Xalapa; un diagnóstico de la oferta y potencialidad musical y enfatizamos que la cultura y creatividad en la música impulsan el desarrollo. Concluimos que Xalapa puede obtener el título de Ciudad Creativa de la Música.

Palabras Clave: Xalapa;

Desarrollo; Ciudad Creativa;

Música; Infraestructura Cultural;

Abstract - The creative and cultural bases of the city of Xalapa, Veracruz can be used and make Xalapa a Creative City of Music (UNESCO). We propose a strategy of development between the government, companies, and society to create a creative industry. In this paper, we present the economic, social and cultural context of Xala- pa; a diagnostic of the musical offer and potential, with the emphasis on how the musical culture and creativity boost development. We conclude that Xalapa can obtain the title of a Creative City of the music.

Keywords: Xalapa; Development; Creative City; Music; Cultural infrastructure;

\section{Xalapa COMO CIUDAD \\ CREATIVA DE LA MÚSICA: PROPUESTA DE DESARROLLO}

En el año 2004, la Organización de las Naciones Unidas para la Educación, la Ciencia y la Cultura (UNESCO por sus siglas en inglés) creó lo que denominó "Red de ciudades creativas" cuyo objetivo es "...posicionar la creatividad y las industrias culturales en el centro de su plan de desarrollo local y cooperar activamente a nivel internacional en la materia"1. Es decir, aprovechar las

1. https://bit.ly/2kRCING

Interconectando Saberes, 2019

ISSN: 2448-8704
Fecha de Recepción: 14 de noviembre de 2018

Fecha de Aceptación: 07 de mayo de 2019

Fecha de Publicación: 30 de junio de 2019 
bases creativas y culturales existentes en cada ciudad y así poder lograr que se genere un círculo virtuoso de desarrollo que involucre a la sociedad y a la economía. El impulso de dicha Red es favorecer a las empresas creativas de las ciudades a través de la cooperación entre gobierno, empresas y sociedad para formar una industria creativa en cada una de las siete categorías propuestas, a saber: Artesanía y artes populares, Arte digital, Diseño, Literatura, Gastronomía, Cine y Música.
Actualmente, la UNESCO tiene registradas 180 ciudades creativas alrededor de 72 países, de las cuales 12 fueron nombradas "Ciudad de la Música" (Ver tabla 1). Desde el año 2015, México cuenta con seis "ciudades creativas", Puebla y el Estado de México son Ciudades Creativas del Diseño, San Cristóbal de las Casas es Ciudad Creativa de la Artesanía y el Arte Popular, Ensenada es Ciudad Creativa de la Gastronomía, Guadalajara es Ciudad Creativa Digital, y Morelia es la primera denominada como Ciudad de la Música.

\section{Tabla 1. Ciudades Musicales}

1.- Almaty (Kazakhstan) - Music

2.- Amarante (Portugal) - Music

3.- Auckland (New Zealand) - Music

4.- Brno (Czechia) - Music

5.- Chennai (India) - Music

6.- Daegu Metropolitan City (Republic of Korea) - Music

7.- Frutillar (Chile) - Music

8.- Kansas City (United States of America) - Music

9.- Morelia (Mexico) - Music

10-Norrköping (Sweden) - Music

11-Pesaro (Italy) - Music

12-Praia (Cabo Verde) - Music

Fuente: Ciudades creativas UNESCO. Disponible en: https://en.unesco.org/creative-cities/events/64-cities-join-unesco-creative-cities-network 
Los principales requisitos que la UNESCO establece para que una ciudad sea catalogada como Ciudad Creativa de la Música, independientemente de que las propias ciudades puedan agregar algunos específicos, son los siguientes ${ }^{2}$ : a) Las ciudades candidatas deben presentar una candidatura que demuestre claramente su determinación, compromiso y capacidad para contribuir a los objetivos de la Red; b) Contar con centros reconocidos de creación y de actividad musical; c) Organizar festivales y otras manifestaciones a escala nacional o internacional; d) Promover la industria musical bajo todas sus formas; e) Contar con conservatorios, academias de música e institutos de educación superior especializados en música; f) Tener instituciones no formales de educación musical, como coros y orquestas de aficionados; g) Poseer plataformas nacionales e internacionales dedicadas a géneros musicales originales $y / 0$ al conocimiento de otros tipos de música; h) Disponer de espacios culturales adaptados para practicar y escuchar música, como auditorios al aire libre; y finalmente, i) Presentar un plan de acción realista de 4 años con proyectos, iniciativas o políticas concretas para alcanzar sus objetivos.

Por lo anterior, el objetivo de este artículo es presentar las potencialidades y fortalezas con las que cuenta la ciudad de Xalapa para que, a propuesta del Ayuntamiento Municipal, sea considerada por la UNESCO como una Ciudad Creativa de la Música.

\section{CONTEXTO SOCIAL,} ECONÓMICO Y CULTURAL DE

\section{XALAPA}

La ciudad de Xalapa-Enríquez es reconocida a nivel nacional e internacional como un centro cultural importante del Estado de Veracruz de Ignacio de la Llave. "[Con]...una larga historia en el apoyo a las artes, y a la música en particular... desde la clásica y el jazz hasta el rock y heavy metal...músicas regionales, sobre todo el son jarocho." (Kohl, 2018, p.17). Actualmente es la capital del Estado con una población de 480, 841 habitantes (INEGI, 2015).

2. Véase: https://bit.ly/2KKQsZL 
El grueso de la población de la ciudad de Xalapa la integran personas de entre 15 y 64 años en un total de 301,266 . Quizá esto se deba a que es la sede de un clúster universitario y de estudios superiores con 71 instituciones que incluyen tres universidades y cuatro institutos públicos; respecto de las instituciones privadas existen 19 universidades, 20 institutos, 23 Centros de Estudios Superiores, un Consorcio y el Colegio de Veracruz, todos registrados ante la Secretaría de Educación del Estado de Veracruz. Con esta infraestructura ofrece el $15.4 \%$ de los servicios educativos estatales y otorga empleos al $14 \%$ de la población total estatal en esta actividad. El nivel de escolaridad indica que el $97 \%$ de su población tiene algún tipo de instrucción escolar desde el primario hasta el universitario (INEGI, Censo 2010).

De acuerdo con el ordenamiento de los sectores económicos establecidos en el Sistema de Clasificación Industrial de América del Norte (SCIAN 2010), al sector de actividades terciarias corresponde, entre otras, los servicios relacionados con la recreación y comprende dos sectores: (sector 71) servicios de esparcimiento culturales y deportivos, y otros servicios recreativos; y (sector 72) servicios de alojamiento temporal y de preparación de alimentos y bebidas. En el sector 71, Xalapa ocupa a 756 personas y en el sector 72 a 10,158 personas. Por lo anterior, la ciudad de Xalapa tiene una concentración muy alta en actividades de servicio comparada con las otras ciudades medias del Estado.

El Censo de Población y Vivienda 2010, cataloga a Xalapa como una ciudad Media porque registró asentamientos humanos de más de 100 mil habitantes. Así se posicionó como la segunda en importancia en el Estado de Veracruz, después de la ciudad de Veracruz y entre ambas concentran el $48 \%$ del total de la población de las 8 ciudades Medias que tiene el Estado (además de 19 ciudades intermedias), con una tasa de crecimiento promedio anual de su población de $1.83 \%$, superior al global estatal de $0.45 \%$. 
Las ciudades Medias del Estado de Veracruz tienen economías de mayor tamaño, irradian la importancia de sus servicios y de su oferta laboral. En particular, Xalapa aporta al Producto Interno Bruto del Estado de Veracruz (PIBE) el $4.2 \%$ con una producción municipal (PIBM) de 11,298 millones de pesos anuales. Los servicios de esparcimiento y recreación, como grupo de actividad, contribuyó con el PIBE en un $11 \%$, con una tasa de crecimiento entre 2004 y 2009 del $23 \%$ dando empleo durante este periodo a casi $11 \mathrm{mil}$ trabajadores. Xalapa está considerada como una ciudad con rezago social muy bajo $(-1.34)$ con un Producto Interno Bruto por Persona de 26,729 pesos y un salario promedio mensual de 6,303 pesos.

En cuanto a la oferta turística y cultural, Xalapa cuenta con museos donde destaca el Museo de Antropología de Xalapa, considerado como el segundo de mayor valor arqueológico en México ${ }^{3}$, después del Museo Nacional de Antropología de la ciudad de México. Además, están el Museo Casa de Xalapa,
Museo Interactivo de Xalapa, Museo del Bombero, Museo de la Fauna y Museo de San Rafael Guízar y Valencia, entre otros.

La ciudad xalapeña cuenta, además, con la infraestructura y atractivos culturales y naturales como salas de concierto y foros para actividades culturales, tales como: Teatro del Estado Ignacio de la Llave, Teatro La Caja, Foro Torre Lapham, Centro Cultural Casa del Lago, Foro La Astilla, Centro Cultural Ágora de la Ciudad, Café-Teatro Tierra Luna, espacio cultural Jardín de las Esculturas y el Centro Recreativo Xalapeño ${ }^{4}$. Además, los auditorios: Auditorio de la Benemérita Escuela Normal Veracruzana Enrique C. Rébsamen, Sala de Conciertos Tlaqná de la Universidad Veracruzana (UV), Auditorios de las diferentes entidades académicas de la UV, Auditorios de las diferentes secciones sindicales, la Sala Tajín y el auditorio del Instituto Superior de Música del Estado de Veracruz.

3. Véase la página: https://bit.ly/2FEomek donde se hace tal afirmación.

4. Consultar: https://bit.ly/2RJuDKw 
También cuenta con galerías para exposiciones y espectáculos -donde se programan exposiciones temporales, distintas expresiones de arte para ofrecer al público diferentes propuestas de calidad a lo largo del año-, entre las que sobresalen: la Pinacoteca de la Ciudad Diego Rivera, Galería del Ágora de la Ciudad, Galería del Centro Recreativo Xalapeño, Galería de Arte Contemporáneo, Galería Ramón Alba de la Canal, Galería Marie Louise Ferrari, Galería Curiel, y el Centro Cultural Fridarte. Sobresalen los espacios para conciertos, tales como: el Museo de Antropología, el Campus para la Cultura, las Artes y el Deporte de la UV, la Unidad de Servicios Bibliotecarios y de Información (USBI), Unidad de Artes de la UV, Cine Club Aula Clavijero y el Instituto de Artes Plásticas.

Cabe mencionar que la actividad editorial de la UV reflejada en la Feria Internacional del Libro Universitario (FILU) en Xalapa, junto con los diversos Festivales, por ejemplo: El Festival Folklórico de Veracruz Miguel Vélez Arceo, la Cumbre Tajín, el Festival Nacional de Títeres de Veracruz Sergio Peregrina
Corona, el Festival Internacional JazzUV, por mencionar algunos; además de Encuentros, Coloquios y Congresos, han permitido que Xalapa se convierta en un centro de atracción para escritores, poetas, decimeros, músicos y pintores provenientes de varias partes de la República Mexicana y resto del mundo. Así como personajes destacados en el teatro, las artes, la música y las humanidades.

Aunado a lo anterior, embellecen a la ciudad de Xalapa, parques y jardines históricos, temáticos y recreativos, donde resalta el Jardín Botánico Francisco Javier Clavijero del Instituto de Ecología, A.C., así como corredores de librerías, bibliotecas y el Estadio Xalapeño Heriberto Jara Corona que puede albergar a 12 mil personas; también ostenta dos palacios de los Poderes Legislativo y Ejecutivo.

Destacamos que, por su semejanza cultural y administrativa con la ciudad de Atenas, también se le conoce a la ciudad de Xalapa como la "Atenas Veracruzana". 


\section{OFERTA XALAPEÑA POR Y}

\section{PARA LAS ARTES}

Desde 1944 la UV se dio a la tarea de formar profesionalmente a los músicos, en 1976 creó la Facultad de Música $^{5}$, donde actualmente se imparten dos licenciaturas: la licenciatura en Música con perfiles de Interpretación, Creación e Investigación y Musicología; y la licenciatura en Educación Musical con perfiles de Música, Docencia e Investigación. Además de dos maestrías: una en Estudios Musicales y otra en Música. Esta última fue el primer posgrado en su tipo de México ${ }^{6}$ y pertenece al Padrón Nacional de Posgrados de Calidad del Consejo Nacional de Ciencia y Tecnología (CONACyT-PNPC).

En 1973 la UV creó la Facultad de Artes Plásticas con el objetivo de formar artistas profesionales con criterio artístico-estético, a través de tres licenciaturas, a saber: Artes Visuales, en Fotografía y en Diseño de la Comunicación Visual. En 1975, instituyó el Área de Artes y con ella la Facultad de Danza -Licenciatura en Danza Contemporá- nea y Enseñanza de las Artes-, misma que fue la primera Institución de Educación Superior pública con enseñanza profesional de la Danza en todo el territorio nacional. Además, existen las facultades y licenciaturas de Artes Plásticas, Danza, Música, Teatro, y el Centro de Estudios de Jazz, así como los estudios de posgrado de Maestría en Artes Escénicas y la Maestría en Música, suscritos en la Dirección General del Área Académica de Artes.

Es importante considerar también a los Talleres Libre de Arte de la UV, dedicados a la educación no formal, cuyo objetivo es despertar el interés por la práctica de las Bellas Artes en la comunidad.

Otra oferta musical a destacar, son los Centros de Iniciación Musical Infantil ubicados en la ciudad de Xalapa -y ciudad de Veracruz-; así como el Centro de Estudios de Jazz de la UV. Este último respalda y fomenta que sus alumnos formen grupos musicales para que desarrollen habilidades al tocar en conjunto $y$

5. Véase: https://bit.ly/2YvkCDz

6. Consultar en: https://bit.ly/2Xla86g 
frente al público; cuenta con siete agrupaciones profesionales: Hilvana, Adal Pérez Cuarteto, Blah Blah Jazz Trío, Prix Bocanegra Cuarteto, Marcelo Lara Quinteto, Rendón Sextet y Zenda. Con interpretaciones que van desde el jazz tradicional proveniente de la música afroamericana, free jazz, soul, folk, bossa nova, reggae, rock y música latinoamericana y contemporánea. Desde el año 2008, este Centro de Estudios realiza cada año el Festival Internacional de Jazzuv, que incluye conciertos, clases maestras, talleres y sesiones de improvisación (jam sessions), con figuras destacadas nacionales e internacionales.

Cabe destacar otras fortalezas, ya que la Dirección General de Difusión Cultural de la UV tiene a su cargo 18 grupos musicales profesionales y tres solistas, entre ellos: Xalli Big Band, grupo Orbis Tertius, el Coro de la UV, el Dueto DelgadoAragón, Dueto Coincidencia, Ensamble Clásico de Guitarras, el Mariachi Universitario, la Orquesta Tradicional Moscovita, Grupo de Música Folklórica Nematatlin, Orquesta de Salsa, Orquesta Universitaria de Música Popular, Orquesta Sinfónica de Xalapa, Grupo Tlen
Huicani, Tlen Huicani Maderas, Grupo Tlayoltiyane, Ronda Jazz, Ballet Folklórico, Ballet Folklórico del Puerto de Veracruz. Así como los solistas: Citlalli Guevara pianista, Armando Mora cantante de ópera y Sandra Lorh cantante de bolero y balada. Estos grupos y cantantes han sido reconocidos a nivel nacional e internacional y cuya trayectoria da realce y calidad artística a la ciudad de Xalapa.

Por otro lado, el gobierno del Estado de Veracruz a través de la Secretaría de Educación creó en 1995, lo que actualmente se conoce como el Instituto Superior de Música del Estado de Veracruz, el cual se ubica en un edificio construido exprofeso para la enseñanza y práctica musical. Cuenta con diez aulas, 24 cubículos insonorizados y climatizados, un foro abierto, auditorio con excelente acústica y laboratorio de teclados, además del equipamiento instrumental necesario para facilitar el aprendizaje. Con sus 21 años de existencia ha creado una orquesta sinfónica juvenil, una banda, un coro infantil y uno de adultos. Cada año ofrece talleres, clases magistrales, así como dos temporadas de conciertos de músi- 
ca de cámara, en la que participan maestros y alumnos. Además, ofrece tres licenciaturas: en Piano, en Ejecución de Instrumentos y en Guitarra.

\section{ECONOMÍA CREATIVA Y Ciudades CReativas}

Para la UNESCO la economía creativa (también llamada economía naranja) está relacionada con todos aquellos procesos productivos que en su eje involucran algún proceso cultural (Buitrago \& Duque, 2013). Para la Conferencia de las Naciones Unidas sobre Comercio y Desarrollo (UNCTAD por sus siglas en inglés) en su informe sobre Economía Creativa 2010, la define como "La economía creativa comprende las áreas del arte, negocios y conectividad, impulsando la innovación y nuevos modelos de negocio." (p. 22).

En el caso de la música, la conectividad digital ha quitado los límites para su comercialización y distribución alrededor del mundo, expandiendo así las industrias culturales que impulsan el crecimiento económico a través de la creación de valor y son elementos clave del sistema de innovación de toda la economía porque trascienden el campo de las artes e incluyen el potencial económico de actividades que antes no se consideraban económicas como, por ejemplo, el quehacer reciente de "pinchar" música que hacen los disc-jockey y que les genera ingresos anuales de millones de dólares, como los 63 millones que ganó el sueco Calvin Harris, considerado por la revista Forbes 2016 como el mejor DJ pagado del mundo, cuyo cobro por espectáculo es de 400 mil dólares.

El Instituto Nacional de Estadística y Geografía (INEGI) implementó por primera vez en el 2012 la medición de la cultura a través de la Cuenta Satélite de la Cultura de México ${ }^{7}$. Para el 2016 esta Cuenta expuso que nuestro sector cultural participó con el $3.3 \%$ del PIB, superando a Canadá $(3.1 \%)$ pero debajo de Estados Unidos (4\%) y España $(4.3 \%)$. Es importante subrayar que nuestro país carece de estadísticas e indicadores en el sector cultural, inclusive éste es uno de los sectores menos atendidos por el INEGI.

7. La implementación de esta Cuenta cumple con las recomendaciones internacionales de la UNESCO, del Convenio Andrés Bello (CAB) y de la Organización Mundial de la Propiedad Intelectual (WIPO por sus siglas en inglés). 
Para conocer qué tanto se aprovecha la cultura en México, Ernesto Piedras (2014) creó el índice compuesto de Capacidades y Aprovechamiento Cultural de los Estados (ICACE $=100$ puntos media nacional) ${ }^{8}$, el cual mide de manera combinada la demanda, la oferta y la infraestructura cultural que tiene cada Estado de la República Mexicana (CONACULTA, 2010). El Estado de Veracruz presenta una alta capacidad y aprovechamiento cultural con un alto consumo de bienes y servicios culturales por arriba de la media nacional con 109.1 puntos. En los subíndices de infraestructura tiene 149.3 puntos, con una demanda de 108.5 puntos y el sub-índice de oferta cultural es de 69.4 puntos. Estos datos muestran que Veracruz cuenta con infraestructura satisfactoria y altas audiencias, pero tiene baja oferta de bienes y servicios culturales.

\section{BENEFICIOS PARA XALAPA DE SER UNA CiUdAD CREATIVA}

En octubre del 2017, el Ayuntamiento de la ciudad de Morelia dio a conocer que la UNESCO la aprobó como Ciudad Creativa de la Música $^{9}$, por la amplia gama de festivales, cientos de músicos e instituciones académicas con las que cuenta junto a la recomendación que extendieron la Escuela de Música de la UNAM y la Escuela de Bellas Artes. Con este nombramiento, el municipio se comprometió a invertir seis millones de pesos, cada año de su administración, para crear 6 proyectos, entre los que se encuentran: El Centro de Documentación y Estudio de la Música, Coros y Orquestas musicales; EI Centro de Estudios de Música para el Cine; y, celebrar el primer Simposio de Industrias Creativas. Además de incentivar los festivales musicales ya establecidos. Este presupuesto se suma a los $15 \mathrm{mi}$ llones de pesos que la ciudad recibe del Congreso de la Unión por ser Ciudad Patrimonio Cultural de la Humanidad. Gracias a esta designación, Morelia se unirá a las Ciudades Creativas para solicitar recursos adicionales que le permitan consolidarse como un destino turístico con más posibilidades culturales.

8. Para consultar el texto ir a: https://bit.ly/2xjlsaf

9. Publicado en el sitio Cambio de Michoacán, en: https://bit.ly/2Jfk5iq 
Así mismo, el nombramiento de ciudades creativas permite también ingresar a una Red como miembro de la UNESCO para implementar estrategias y planes de apoyo realistas y concretos para el crecimiento de actividades creativas $y$ culturales en el largo plazo (o proyectos de 4 años), donde los postulantes se comprometen a participar con un enfoque orientado al futuro para alcanzar los objetivos de la Red. Adem's se comprometen a diseñar políticas culturales, de derechos de autor, y obtener recursos por la actividad turística, tal y como lo hacen Canadá, Italia, Portugal, Grecia, Inglaterra, Francia, entre otros países (Autissier, 2013).

Otro beneficio de pertenecer a una Red Creativa es que, en el marco de la aplicación de la agenda 2030 sobre el Desarrollo Sostenible, las ciudades miembro pueden mostrar su cultura como motor de sostenibilidad, utilizando una plataforma internacional, ventana que sirve para promoverse e incrementar el turismo o venta de bienes y/o servicios creativos, además de fomentar el intercambio de capacitación técnica, formación empresarial de los agentes culturales y compartir las experiencias y conocimientos con otros espacios culturales del mundo. Tal y como hoy lo hace Morelia en: www.moreliacreativecity.org/.

\section{CONCLUSIONES}

Mostramos que la ciudad de Xalapa reúne los requisitos más importantes para ser considerada como una Ciudad Creativa de la Música, toda vez que cuenta con los elementos y potencial para generar un círculo virtuoso sustentado formal e institucionalmente por sus espacios culturales, facultades, cientos de músicos y grupos artísticos musicales y profesionales tanto de la Universidad Veracruzana como del gobierno estatal y municipal, así como toda la gama de espectáculos, ferias y festivales de alcance nacional e internacional.

Beneficiarse con el título de Ciudad Creativa de la Música por parte de la UNESCO, incentivará al Ayuntamiento de Xalapa, tal y como lo hizo el de Morelia, a diseñar una política cultural particular y realista, que impulse a la economía creativa 
xalapeña desde un marco legal que proteja y beneficie a las instituciones, artistas y creadores de la cultura, así como satisfaga sus necesidades de información, difusión y generación de empleos; por ello es que se considera que esta es una propuesta factible de desarrollo para la ciudad de Xalapa.

\section{REFERENCIAS}

Autissier, Anne-Marie, (2013). Las políticas culturales en Europa: desafíos sociales e identitarios. Disponible en: https://amnis.revues.org/2050

Buitrago, R. e I., Duque., (2013). La Economía Naranja: una oportunidad infinita. Washington: Banco Interamericano de Desarrollo.

Consejo Nacional para la Cultura y las Artes, (2010). Encuesta Nacional de Consumo y Prácticas Culturales 2010 para el Estado de Veracruz. Disponible en: http://www.cultura.gob.mx/encuesta_ nacional/\#.WIwxlaawKdM

Consejo Nacional para la Cultura y las Artes, (2010). Atlas de Infraestructura y Patrimonio Cultural de México, Disponible en: http://sic.gob.mx/atlas2010/fo/ATLAS -1a-parte.pdf

Instituto Nacional de Estadística y Geografía (INEGI), (2015). Encuesta Intercensal 2015. Disponible en: http://cuentame.inegi.org.mx
Instituto Nacional de Estadística y Geografía (INEGI), (2010). Censo de Población y Vivienda 2010.

Principales resultados por localidad. Disponible en:

http://www.inegi.org.mx/est/contenid os/proyectos/ccpv/cpv2010/iter_2010 .aspx

Instituto Nacional de Estadística y Geografía (INEGI), (2009). Censo Económico 2009. Disponible en: www.inegi.org.mx/est/contenidos/esp anol/proyectos/censos/ce2009/

Instituto Nacional de Estadística y Geografía (INEGI), (2015). Sistema de Cuentas Nacionales de México. Cuenta satélite de la cultura de México 2013. Año base 2008., Disponible en:

https://www.inegi.org.mx/app/bibliote ca/ficha.html?upc=702825074241

Instituto Nacional de Estadística y Geografía (INEGI), (2013). Sistema de Clasificación Industrial de América del Norte, México: SCIAN 2013. en:

https://www.inegi.org.mx/app/bibliote ca/ficha.html?upc=702825051693

Kohl, R., (2018), ¿Músico pagado toca mal son? Unas miradas al mercado laboral del son jarocho. México: Instituto Veracruzano de la Cultura.

Piedras, E., (2014), ¿Cuánto vale la Cultura? Medición de las Industrias Culturales y Creativas en México en: http://politicasculturales.com.mx/201 4/cuanto-vale.html

Piedras, E., (Coordinador), (2011). Índice de Capacidades y Aprovechamiento Cultural de los Estados ICACE. Disponible en: www.nomismae.net/nomismae_ok/p df/Nomismae-ICACE_2011.pdf 
Piedras, E., (2012). La cultura es necesaria, se justifique o no en lo económico. El Informador.

Disponible en:

http://www.informador.com.mx/suple mentos/2012/388402/6/la-cultura-esnecesaria-se-justifique-o-no-en-loeconomico.htm

Organización de las Naciones Unidas para la Educación, la Ciencia y la Cultura (UNESCO), (1982). Declaración de México sobre las Políticas Culturales, Conferencia Mundial sobre las políticas culturales. 26 de julio- 6 de agosto de 1982. Disponible en: http://portal.unesco.org/culture/es/file s/35197/11919413801mexico_sp.pdf /mexico_sp.pdf

Organización de las Naciones Unidas para la Educación, la Ciencia y la Cultura (UNESCO), (1998). Plan de Acción de Políticas Culturales para el Desarrollo, 1998. Disponible en: https://unesdoc.unesco.org/ark:/4822 3/pf0000116393_spa

Organización de las Naciones Unidas para la Educación, la Ciencia y la Cultura (UNESCO), (2019). Creative Cities Network. Disponible en https://es.unesco.org/creativecities/content/ciudades-creativas 